\title{
Brain Stimulation by Direct Current Can Help Improvement of Nicotine and Alcohol Addiction in Nepal
}

\author{
Razia Sultana ${ }^{1}$, Shahid Bashir ${ }^{2}$, Shinawar Waseem Ali ${ }^{1}$, Ghulam Murtaza," \\ ${ }^{1}$ Institute of Agricultural Sciences, University of the Punjab, Pakistan \\ ${ }^{2}$ Department of Physiology, College of Medicine, King Saud University, Saudi Arabia \\ ${ }^{3}$ Department of Neurology, College of Medicine, King Saud University, Saudi Arabia
}

Copyright $\mathrm{C} 2017$ by authors, all rights reserved. Authors agree that this article remains permanently open access under the terms of the Creative Commons Attribution License 4.0 International License

\begin{abstract}
Nicotine and alcohol are highly addictive substances, which make their consumers difficult to get rid of them. These substances are the risk factors for many diseases including stroke, pulmonary and cardiovascular diseases. In Nepal, in spite of measures taken by the government and non-governmental organizations to intervene their consumption, prevalence of these drugs and dependency of people on them are quite high. Introduction and implementation of new and inexpensive technology such as transcranial direct current stimulation can help people get rid of addiction.
\end{abstract}

Keywords Transcranial Direct Current Stimulation, Addiction, Nicotine, Alcohol, Nepal

\section{Introduction}

Addiction is defined as a primary, chronic disease of brain reward, motivation, memory and related circuitry. Despite adverse consequences, people get engage in seeking an addictive substance or activity. It is often debated that whether addiction is a personal choice or biological vulnerability. Initially, people take addictive substances voluntarily and their continuous use impairs brain function. Addicted persons lose self-control over their addictive behavior. Persons with chronic stress, social anxiety, and depression start using drugs to lessen feelings of distress. Moreover, people with genetic vulnerability and those who start taking abused drugs during early adolescence are at higher risk of dependency of addictive substance. Recent studies have presented evidence that addictive substances activate various reward circuits in the brain and thus exercise their initial reinforcing effects [1].

Nicotine and alcohol are known as highly addictive substances. Once initiated, they make their consumers difficult to get rid of them. Alcohol has been around since time unknown. It is mind-alerting drug with many stimulating effects. It is consumed by people of every age group and both genders alike [2]. It is considered as one of the most dangerous drugs for human. Consuming alcohol first time people do not think to become its addicted as it has milder effects compared to nicotine. With the passage of time, people crave more of the alcohol to feel the same high effect as earlier. People become alcoholic through two ways; positive and negative reinforcement methods. In positive reinforcement, people consume alcohol according to the environmental situation (e.g., alcohol-induced euphoria). In negative reinforcement, people use alcohol without caring about its negative outcome. Addicted people when try to abstain from nicotine and alcohol consumption may experience anxiety, irritability, and dysphoria. These symptoms make forbearance difficult and a relapse possible [3]. The reward pathways of the brain are not always under voluntary control. In addicted persons, the neurobiology of the brain will change permanently [4]. Nicotine and alcohol use are closely related behaviors. Thus, people who drink alcohol are more like to consume nicotine and vice versa. Moreover, patients diagnosed with addiction of one substance are commonly diagnosed with addiction of another substance. Although, smoking rate in general population has declined over the past decades, smoking rate among alcohol addicted persons is still high [5]. Concurrent use of nicotine and alcohol has detrimental effects not only on individuals but also on society. It has become clear that their heavy consumption is a risk factor for many leading causes of death worldwide. About five million deaths per year occur worldwide due to nicotine consumption, which are expected to project to more than eight million by 2030 [6].

Nicotine and alcohol are public health concerns as their consumption is associated with physical ailments such as cancer, pulmonary and cardiovascular diseases, birth disorders, cirrhosis etc. [2]. Due to their increased use and side effects, attention should be paid to their consumers particularly who are dependent on both. Motivation and 
education are key factors in controlling their use. Clinical studies have proved that cessation of nicotine and alcohol consumption is not possible without the will of addicted persons. Addicted persons become depressed when they stop consuming nicotine and alcohol. Antidepressant treatment is recommended for the patients when depression lasts 2-4 weeks of cessation of drug [7]; for alcoholism and drug treatment see [2]. In fact, over the past decades, both researchers and policy makers have taken addiction as a serious concern. Several new methods have been introduced and implemented to interrupt this disease. However, consumption of alcohol and tobacco products is still high particularly in South Asia including Nepal $[8,9]$. The focus of this review is to highlight the effects of heavy consumption of nicotine and alcohol in Nepalese population and how new techniques such as brain stimulation by direct current can help people get rid of their addiction.

\section{Factors Contributing to Nicotine and Alcohol Consumption}

Different factors are linked with use/co-use of nicotine and alcohol. One study has demonstrated that smoking and alcohol use in adolescents is associated with shared environmental features, for instance peer influence. However, in adults, this behavior was linked with genetic factors [10]. Neurobiological mechanisms may contribute for co-use of two or more drugs. Ability of one drug to increase the effect of other drug can mediate this relationship. It has been demonstrated that co-use of two drugs may influence the nerve cell functions. Concurrent use of two drugs may increase the qualitative reward effect that is not achievable by either drug alone [11]. Psychosocial factors such as sensation seeking, impulsivity, compulsiveness, and neuroticism (i.e., trait anxiety) contribute to consumption of alcohol and nicotine [12]. People under certain conditions get engage in smoking once again. For instance, in parties and bars people normally smoke along with drinking alcohol [13]. Another reason for using nicotine and alcohol is their additive or synergistic analgesic effects [14]. Moreover, concurrent use of nicotine and alcohol may be due to counteracting mechanisms. Harmful effects of alcohol such as cognitive impairment, intoxication, sedation etc., are alleviated by nicotine [15]. About $40-80 \%$ of addictive disorders are due to genetic factors [16], which suggest genetic vulnerability for nicotine-alcohol co-morbidity. In nicotine and alcohol dependency, genetic factors account for about $50 \%[17,18]$, however this number may vary according to gender, region, and culture. Moreover, contribution of genetic factors in nicotine-alcohol co-morbidity is equal in males and females [19]. Genetic studies of addiction demonstrate that many genes act and modify various functions such as enzymatic activity, protein translation, receptor functions, and transcriptional regulation that are involved in the reinforcement of addictive compounds [16]. Animal studies show that modifications in genetic makeup can result in changes in sensitivity to drugs and release of dopamine (DA) [20]. Another consideration is that epigenetic changes also result in addictive behaviors. A previous study describes that changes in gene expression in neurons can lead to changes in behaviors including addiction [21].

\section{Brain Areas Involved in Addiction}

Following brain regions are involved in the addictive behavior.

Amygdala: It regulates memory consolidation for emotionally arousing events. For instance, in rodents, selection of a favorite and specific cage was lost with the ablation of amygdala [22].

Anterior cingulate: Involved in emotional self-control and adaptive response to changing conditions [23].

Bed nucleus of the stria terminalis (BNST): It is considered part of the extended amygdala. It is sensitive to DA stimulation [24].

Dorsolateral prefrontal cortex (DLPFC): It controls and regulates cognitive activities including the sequencing of events, planning, and the selection of objectives [25].

Hippocampus: Involved in acquisition of new information and formation of new memories about personally experienced incidents [26].

Insular cortex: Involved in processing of pain and consciousness. It receives visceral, olfactory, gustatory, and other somatosensory information [27].

Orbitofrontal cortex (OFC): Critical for impulsivity and decision making. The medial OFC (ventromedial cortex) with connections to the hippocampus and cingulate is important for assessing the familiarity or "rightness" of a situation and in integrating outcome expectancies. The lateral OFC in association with amygdala and insula is linked with the suppression of previously rewarded responses [28]; for further details see [29].

\section{Reward Signaling in the Brain}

Nicotine and alcohol activate reward pathways to enhance the rewarding effects. It is reported that nicotine dependence is related to the conditioned aspects of the environment [30]. However, recent studies have demonstrated that nicotine produces its reinforcing and addictive effects by activating dopaminergic (DA) pathway $[31,32]$. In a functional magnetic resonance imaging (MRI) study, in nicotine-deprived smokers, activation of mesolimbic DA reward circuits after exposure to smoking-related images was higher compared with neutral images [33-36]. Moreover, decrease in DA D1 receptor (D1R) binding in the ventral striatum was described in cigarette smokers in a positron emission tomography (PET) study [37]. DA neurons are located in the ventral tegmental area (VTA) and nucleus accumbens (NAc). Activation of NAc has a link with the processing of reward-related stimuli 
and regulation of reward-induced addiction [38, 39]. In animal studies, dysfunctioning of NAc reduces drug-seeking behavior by decreasing the motivational effect of the conditioned stimulus [40, 41]. Takahashi $\mathrm{H}$ et al. [4] demonstrated correlation of DA release in the ventral striatum following nicotine application with the degree of nicotine dependence. In addition, previous findings present evidence of the contribution of ventral striatum-nucleus accumbens (VS-NAc) to the experience of subjective cigarette craving [33-35].

Alcohol is a powerful drug that activates several neurological pathways and brings about prominent modifications in the brain. The dopaminergic, serotoninergic, $\gamma$-amino butyric acid (GABA) and glutamate pathways are few examples of such pathways that are affected by alcohol.

DA pathway: DA, a neurotransmitter, is involved in a circuit called the mesolimbic system, which projects from the brain's VTA to the NAc. This circuit is involved in incentive motivation. DA has a marked role in the incentive motivation associated with acute alcohol intoxication. Alcohol consumption can be intervened by injecting DA antagonist into the NAc $[42,43]$. Furthermore, it has been observed that production of DA in NAc is associated with the alcohol consummation and anticipated availability [43].

Serotonin pathway: Production/availability of serotonin neurotransmitter (also known as 5-hydroxytryptamine (5-HT)) has a strong link with the level of alcohol consumption. In animal studies, during alcohol abstinence, serotonin production in NAc was depressed [44].

GABA pathway: Action of alcohol increases activity of GABA, which is a major inhibitory neurotransmitter in the brain. The use of alcohol is suppressed by substances that interfere with the actions of the GABA receptors in the NAc, ventral pallidum, bed nucleus of the stria terminalis and amygdala; for further details about role of GABA in the motivational effects of alcohol see [45].

Glutamate pathway: Glutamate (a major excitatory neurotransmitter) system is involved in acute reinforcing actions of alcohol. Activity of glutamate is inhibited by alcohol. Acute alcohol consumption results in reduction of glutamate levels in the rat striatum [46]. Glutamate exerts its effects through several receptors including one $\mathrm{N}$-methyl-D-aspartate (NMDA) receptor. A previous report has presented evidence that NMDA receptor antagonists can mimic effects of alcohol [47].

Thus, nicotine and alcohol are involved in dopaminergic neuron firing [48]. Release of DA increases when nicotine and alcohol are administered together [49]. Extra release of DA explains additive rewarding effect and possible phenomenon behind the co-use of nicotine and alcohol. For natural reinforces (food and sex) conditioned stimuli provoke DA signals, which drive the motivation for reward acquisition. DA cells stop firing after consumption of natural reinforces. However, in case of drugs of abuse, DA release will keep increasing during their consumption thus resulting in dependency and compulsive behaviors [50].

\section{Transcranial Direct Current Stimulation (tDCS)}

The brain areas such as DLPFC, OFC, and sub-cortical regions are considered to exert cognitive control over feelings of craving reward related to nicotine and alcohol use. These brains regions are easily accessible and targets of non-invasive stimulation by direct current. Certain studies have demonstrated that tDCS modifies afore-mentioned areas, which results in a reduction of craving rates [51, 52]. tDCS as a technique of non-invasive cortical stimulation has gained renewed clinical importance since 1998 [36, 53]. It generates an electric field in the brain and significantly modulates spontaneous neuronal activity [54]. Initially, studies using tDCS were carried out in healthy subjects. It took several years to consider this technique for therapeutic purposes. First clinical work using this method was published in 2005, where stimulation by a weak current significantly improved motor hand function in stroke patients [55]. Afterwards, about 340 articles have been published demonstrating effects of tDCS in patients with various types of diseases. These days it is being used in healthy people also for cognitive enhancement [56].

A tDCS device consists of an adjustable direct current stimulator and two stimulating anode and cathode electrodes, which are attached to two separate positions on the scalp (either directly or through larger sponge electrodes). After switching on the device, a weak current of $0.5-2.0 \mathrm{~mA}$ passes between the electrodes, diffuses in the brain regions, and modulates neural activity underneath the electrode. The experimental data demonstrate that neurons are sensitive to weak electric fields and low density current is sufficient enough to induce action potential firing changes, synaptic plasticity triggering, or neurogenesis promotion [57]. tDCS modulates brain functions by changing the resting membrane potential of the nerve cells, where the neurons proximal to the anode and cathode are depolarized and hyperpolarized, respectively[58].

\section{Considerations for tDCS Application}

Before administering tDCS, one must consider certain conditions for its safe use and beneficial effects. It is not recommended for subjects with unstable medical conditions and uncontrolled seizures, for instance people with epilepsy [59]. The electrode in tDCS is the place of electrochemical reactions [60]. It should not be in direct contact with the skin [61]. Precise administration of electrodes on the specific brain areas along with adherence to established tDCS protocols is mandatory to avoid skin injury [62-65]. Placement of electrodes depends upon the anatomical features of individuals' heads. Thus, it is important to use suitable methods such as (1) international 10-20 (or 10-5) electrode placement system [66, 67], or another gross anatomical coordinate system [68], (2) neuronavigation 
systems [69-72], and (3) physiology-based placement for proper positioning of electrodes. However, physiology-based placement can only be carried out for motor and other primary cortices [73]. In addition, rate of current flow into the brain is proportional to the size of electrodes employed [74]. In neurophysiological modulations, lasting of current-induced after-effects depends on the duration of stimulation. For instance, in healthy individuals, stimulation of resting motor cortex by tDCS (1 $\mathrm{mA}$ current intensity, electrode size $35 \mathrm{~cm}^{2}$ ) for $4 \mathrm{~s}$ does not induce after-effects [36]. Stimulation for 5-7 min results in lasting of after-effects for $10 \mathrm{~min}$. Furthermore, $9 \mathrm{~min}$ stimulation under the cathode electrode and $13 \mathrm{~min}$ stimulation under the anode result in lasting of after-effects for about $1 \mathrm{~h}$ [36, 75, 76]; for a detailed review see [58]. Further increase of current intensity and/or duration of stimulation will not guarantee relative increase of after-effects but can result in negative effects [77, 78]. It is recommended to use spaced stimulation with intervals $\leqslant 30$ min to achieve relevantly longer-lasting tDCS effects $[78$, 79]. The user should wait at least $48 \mathrm{~h}$ to a week before repeating the tDCS [80].

The persons who are administered with tDCS often complain tingling and itching sensations under the electrodes, headache, and tiredness [63, 81]. Preparing electrodes and skin with saline solution and electrode cream, respectively and ramping up the current intensity can reduce the irritation effects/sensation [82]. The tDCS operator should inform persons who receive tDCS about potential risks and benefits associated with tDCS procedure. He/she should obtain informed consent for the procedure. Moreover, ethical approval should also be obtained from institutional review board when tDCS is used for research purposes [80]. Then, this is the responsibility of tDCS operator to follow the approved protocol only [73]. If children are exposed to tDCS treatment, special consideration should be paid as parameters safely used in adults may have different safety and tolerability conditions $[83,84]$.

tDCS administration in comparison with other methods of brain stimulation is an easy and user-friendly method. Currently, companies for the promotion of tDCS use are organizing certain workshops and courses. Moreover, do-it-yourself (DIY) brain stimulation is going on increasing [85]. Yet, tDCS has not been integrated into the medical graduate and postgraduate education. Its safe application requires comprehensive and systematic training $[73,85]$. The tDCS operator should be well trained and competent in different aspects, which include theoretical background of tDCS, principles and rationale of tDCS application in specific populations, subjects selection, stimulation protocol, safety evidence and safety precautions, preparation and positioning of the electrodes, operation of the tDCS unit, and outcome monitoring and recording; for further details see also [73].

\section{Nicotine and Alcohol Addiction in Nepal and Implementation of tDCS}

Nepal is a low-income landlocked country located between India and China. It has a population of about 26 million people and area of 147,181 square kilometers. In Nepal, nicotine consumption is quite high and its prevalence appears to be increasing from $7.8 \%$ in $2001,9.4 \%$ in 2007 , $13.9 \%$ in $2008,20.4 \%$ in 2011 to $25 \%$ in 2016 [86]. In adult men and women, overall smoking prevalence ranges between $25 \%$ to $73 \%$ and $0.8 \%$ to $60 \%$, respectively in different areas of the country. Children start using tobacco at the age of 13 years. Tobacco consumption is associated with ethnicity, sex, family members and friends using tobacco products [86]. Alcohol as part of culture has been in Nepal since time immemorial with high dependency [9]. Its acceptance in society is so high that its use is common practice even among adolescents and married women of reproductive age $[87,88]$. Initially, it was not considered as a serious problem by the government and social organizations. Presently, this drug is the number one problem in this country. Several people are arrested daily for drinking and rowdy behavior [89]. A 'Matwali' is a person who is allowed to consume alcohol by virtue of his birth. A high percentage of the Nepalese population falls to this category. There are many reasons that account for alcohol consumption in Nepal. People generally believe that alcohol is a remedy for pain, cold, physical tiredness, mental tension, sleeplessness, and social inhibitions. Most of the unskilled and semi-skilled workers consider alcohol drinking necessary for their better working. It is a status symbol for many people. Parties, get-togethers or festivities are incomplete without serving of alcoholic beverages [89].

Despite high nicotine and alcohol consumption, scientific investigations are missing about level of their dependence among their consumers in Nepal. The Government of Nepal and non-governmental organizations (NGOs) have taken number of measures to counter production and supply of tobacco and alcohol. In 2003, Government of Nepal signed World Health Organization Framework Convention on Tobacco Control (WHO FCTC). Moreover, government has also introduced and implemented the Tobacco Product Control and Regulatory Act 2011, Tobacco Product Control and Regulatory Rule 2012 and Directive for Printing and Labeling of Warning Message and Picture in the Box, Packet, Wrapper, Carton, Parcel and Packaging of Tobacco Product, 2011 [86]. NGOs including Nepal Cancer Relief Society (NCRS), Social Services National Co-ordination Council (SSNCC), Drug Abuse Prevention Association of Nepal (DAPAN), St. Xavier's Social Service Centres, and Health Research and Social Development Forum (HERD) have established their branches and mobilized volunteers countrywide to control nicotine consumption. These organizations work to prevent drug consumption and raise awareness about harms linked with alcohol and nicotine consumption. 
However, consumption of afore-mentioned products is still high. On the other hand, smokers themselves try to quit smoking several times but fail to get rid of it. Thus development of effective smoking intervention programs is recommended for Nepalese population [8]. Recent investigations have demonstrated that brain stimulation by direct current can increase resistance to smoking and alcohol. Previous investigations demonstrate that anodal stimulation over the left DLPFC (with cathodal stimulation over the right supra-orbital area) can increase resistance against smoking and alcohol [90-93]. The rate of stroke incidences are increasing in Nepal, which is considered to be associated with nicotine and alcohol uses [94]. Thus, this is the need of hour to introduce and implement latest and cheaper technology such as tDCS in Nepal to help people get rid of addiction of risk factors for many lethal diseases. Moreover, it will reduce the burden of government in spending a lot of money on the management of many diseases.

\section{REFERENCES}

[1] Volkow, N.D. and M. Morales, The Brain on Drugs: From Reward to Addiction. Cell, 2015. 162(4): p. 712-25.

[2] Crocq, M.A., Alcohol, nicotine, caffeine, and mental disorders. Dialogues Clin Neurosci, 2003. 5(2): p. 175-85.

[3] Fowler, I.L., et al., Patterns of current and lifetime substance use in schizophrenia. Schizophrenia Bulletin, 1998. 24(3): p. $443-455$.

[4] Banerjee, N., Neurotransmitters in alcoholism: A review of neurobiological and genetic studies. Indian J Hum Genet, 2014. 20(1): p. 20-31.

[5] Hays, J.T., et al., Response to nicotine dependence treatment in smokers with current and past alcohol problems. Ann Behav Med, 1999. 21(3): p. 244-50.

[6] Mathers, C.D. and D. Loncar, Projections of global mortality and burden of disease from 2002 to 2030. PLoS Med, 2006. 3(11): p. e442.

[7] Greenfield, S.F., et al., The effect of depression on return to drinking: a prospective study. Arch Gen Psychiatry, 1998. 55(3): p. 259-65.

[8] Aryal, U.R., et al., Assessment of nicotine dependence among smokers in Nepal: a community based cross-sectional study. Tobacco Induced Diseases, 2015. 13.

[9] Shrestha, N.M., Alcohol and Drug-Abuse in Nepal. British Journal of Addiction, 1992. 87(9): p. 1241-1248.

[10] Koopmans, J.R., L.J.P. vanDoornen, and D.I. Boomsma, Association between alcohol use and smoking in adolescent and young adult twins: A bivariate genetic analysis. Alcoholism-Clinical and Experimental Research, 1997. 21(3): p. 537-546.

[11] Yue, J., et al., Differential induction of ethanol-metabolizing CYP2E1 and nicotine-metabolizing CYP2B1/2 in rat liver by chronic nicotine treatment and voluntary ethanol intake.
European Journal of Pharmacology, 2009. 609(1-3): p. 88-95.

[12] Mitchell, M.R. and M.N. Potenza, Addictions and Personality Traits: Impulsivity and Related Constructs. Curr Behav Neurosci Rep, 2014. 1(1): p. 1-12.

[13] Brandon, T.H., et al., Postcessation Cigarette Use - the Process of Relapse. Addictive Behaviors, 1990. 15(2): p. 105-114.

[14] Franklin, K.B., Analgesia and abuse potential: an accidental association or a common substrate? Pharmacol Biochem Behav, 1998. 59(4): p. 993-1002.

[15] Ceballos, N.A., Tobacco use, alcohol dependence, and cognitive performance. Journal of General Psychology, 2006. 133(4): p. 375-388.

[16] Goldman, D., G. Oroszi, and F. Ducci, The genetics of addictions: uncovering the genes. Nat Rev Genet, 2005. 6(7): p. 521-32.

[17] Madden, P.A., et al., Smoking and the genetic contribution to alcohol-dependence risk. Alcohol Res Health, 2000. 24(4): p. 209-14.

[18] Sartor, C.E., et al., Common Genetic Contributions to Alcohol and Cannabis Use and Dependence Symptomatology. Alcoholism-Clinical and Experimental Research, 2010. 34(3): p. 545-554.

[19] Han, C., M.K. McGue, and W.G. Iacono, Lifetime tobacco, alcohol and other substance use in adolescent Minnesota twins: univariate and multivariate behavioral genetic analyses. Addiction, 1999. 94(7): p. 981-993.

[20] Cadoni, C., T. Muto, and G. Di Chiara, Nicotine differentially affects dopamine transmission in the nucleus accumbens shell and core of Lewis and Fischer 344 rats. Neuropharmacology, 2009. 57(5-6): p. 496-501.

[21] Tsankova, N., et al., Epigenetic regulation in psychiatric disorders. Nat Rev Neurosci, 2007. 8(5): p. 355-67.

[22] Adolphs, R., What does the amygdala contribute to social cognition? Year in Cognitive Neuroscience 2010, 2010. 1191: p. 42-61.

[23] Allman, J.M., et al., The anterior cingulate cortex - The evolution of an interface between emotion and cognition. Unity of Knowledge: The Convergence of Natural and Human Science, 2001. 935: p. 107-117.

[24] Erb, S. and J. Stewart, A role for the bed nucleus of the stria terminalis, but not the amygdala, in the effects of corticotropin-releasing factor on stress-induced reinstatement of cocaine seeking. Journal of Neuroscience, 1999. 19(20): p. art. no.-RC35.

[25] Ballard, I.C., et al., Dorsolateral prefrontal cortex drives mesolimbic dopaminergic regions to initiate motivated behavior. J Neurosci, 2011. 31(28): p. 10340-6.

[26] Dickerson, B.C. and H. Eichenbaum, The episodic memory system: neurocircuitry and disorders. Neuropsychopharmacol ogy, 2010. 35(1): p. 86-104.

[27] Starr, C.J., et al., Roles of the insular cortex in the modulation of pain: insights from brain lesions. J Neurosci, 2009. 29(9): p. 2684-94.

[28] Elliott, R., R.J. Dolan, and C.D. Frith, Dissociable functions in 
the medial and lateral orbitofrontal cortex: evidence from human neuroimaging studies. Cereb Cortex, 2000. 10(3): p. 308-17.

[29] Adinoff, B., Neurobiologic processes in drug reward and addiction. Harvard Review of Psychiatry, 2004. 12(6): p. 305-320.

[30] Miyata, H. and T. Yanagita, Neurobiological mechanisms of nicotine craving. Alcohol, 2001. 24(2): p. 87-93.

[31] Rice, M.E. and S.J. Cragg, Nicotine amplifies reward-related dopamine signals in striatum. Nat Neurosci, 2004. 7(6): p. 583-4.

[32] Zhang, H. and D. Sulzer, Frequency-dependent modulation of dopamine release by nicotine. Nat Neurosci, 2004. 7(6): p. 581-2.

[33] David, S.P., et al., Ventral striatum/nucleus accumbens activation to smoking-related pictorial cues in smokers and nonsmokers: a functional magnetic resonance imaging study. Biol Psychiatry, 2005. 58(6): p. 488-94.

[34] Due, D.L., et al., Activation in mesolimbic and visuospatial neural circuits elicited by smoking cues: evidence from functional magnetic resonance imaging. Am J Psychiatry, 2002. 159(6): p. 954-60.

[35] McClernon, F.J., et al., Abstinence-induced changes in self-report craving correlate with event-related FMRI responses to smoking cues. Neuropsychopharmacology, 2005. 30(10): p. 1940-7.

[36] Nitsche, M.A. and W. Paulus, Excitability changes induced in the human motor cortex by weak transcranial direct current stimulation. J Physiol, 2000. 527 Pt 3: p. 633-9.

[37] Dagher, A., et al., Reduced dopamine D1 receptor binding in the ventral striatum of cigarette smokers. Synapse, 2001. 42(1): p. $48-53$.

[38] Cardinal, R.N., et al., Emotion and motivation: the role of the amygdala, ventral striatum, and prefrontal cortex. Neurosci Biobehav Rev, 2002. 26(3): p. 321-52.

[39] Parkinson, J.A., et al., Nucleus accumbens dopamine depletion impairs both acquisition and performance of appetitive Pavlovian approach behaviour: implications for mesoaccumbens dopamine function. Behavioural Brain Research, 2002. 137(1-2): p. 149-163.

[40] Hutcheson, D.M., et al., The effects of nucleus accumbens core and shell lesions on intravenous heroin self-administration and the acquisition of drug-seeking behaviour under a second-order schedule of heroin reinforcement. Psychopharmacology (Berl), 2001. 153(4): p. 464-72.

[41] Ito, R., T.W. Robbins, and B.J. Everitt, Differential control over cocaine-seeking behavior by nucleus accumbens core and shell. Nat Neurosci, 2004. 7(4): p. 389-97.

[42] Hodge, C.W., H.H. Samson, and A.M. Chappelle, Alcohol self-administration: Further examination of the role of dopamine receptors in the nucleus accumbens. Alcoholism-Clinical and Experimental Research, 1997. 21(6): p. 1083-1091.

[43] Rassnick, S., L. Pulvirenti, and G.F. Koob, Oral Ethanol Self-Administration in Rats Is Reduced by the Administration of Dopamine and Glutamate Receptor Antagonists into the Nucleus-Accumbens. Psychopharmacology, 1992. 109(1-2): p. 92-98.

[44] Weiss, F., et al., Ethanol self-administration restores withdrawal-associated deficiencies in accumbal dopamine and 5-hydroxytryptamine release in dependent rats. J Neurosci, 1996. 16(10): p. 3474-85.

[45] Koob, G.F., A role for GABA mechanisms in the motivational effects of alcohol. Biochem Pharmacol, 2004. 68(8): p. 1515-25.

[46] Carboni, S., et al., Ethanol prevents the glutamate release induced by N-methyl-D-aspartate in the rat striatum. Neurosci Lett, 1993. 152(1-2): p. 133-6.

[47] Gilpin, N.W. and G.F. Koob, Neurobiology of alcohol dependence: focus on motivational mechanisms. Alcohol Res Health, 2008. 31(3): p. 185-95.

[48] Kleijn, J., et al., Direct effect of nicotine on mesolimbic dopamine release in rat nucleus accumbens shell. Neurosci Lett, 2011. 493(1-2): p. 55-8.

[49] Tizabi, Y., et al., Combined effects of systemic alcohol and nicotine on dopamine release in the nucleus accumbens shell. Alcohol Alcohol, 2007. 42(5): p. 413-6.

[50] Schultz, W., P. Dayan, and P.R. Montague, A neural substrate of prediction and reward. Science, 1997. 275(5306): p. 1593-9.

[51] Goldstein, R.Z. and N.D. Volkow, Dysfunction of the prefrontal cortex in addiction: neuroimaging findings and clinical implications. Nat Rev Neurosci, 2011. 12(11): p. 652-69.

[52] Nestor, L., et al., Differences in "bottom-up" and "top-down" neural activity in current and former cigarette smokers: Evidence for neural substrates which may promote nicotine abstinence through increased cognitive control. Neuroimage, 2011. 56(4): p. 2258-2275.

[53] Priori, A., et al., Polarization of the human motor cortex through the scalp. Neuroreport, 1998. 9(10): p. 2257-2260.

[54] Fritsch, B., et al., Direct current stimulation promotes BDNF-dependent synaptic plasticity: potential implications for motor learning. Neuron, 2010. 66(2): p. 198-204.

[55] Hummel, F., et al., Effects of non-invasive cortical stimulation on skilled motor function in chronic stroke. Brain, 2005. 128: p. 490-499.

[56] Lefaucheur, J.P., A comprehensive database of published tDCS clinical trials (2005-2016). Neurophysiol Clin, 2016. 46(6): p. 319-398.

[57] Francis, J.T., B.J. Gluckman, and S.J. Schiff, Sensitivity of neurons to weak electric fields. J Neurosci, 2003. 23(19): p. 7255-61.

[58] Stagg, C.J. and M.A. Nitsche, Physiological basis of transcranial direct current stimulation. Neuroscientist, 2011. 17(1): p. 37-53.

[59] Fregni, F., et al., A controlled clinical trial of cathodal DC polarization in patients with refractory epilepsy. Epilepsia, 2006. 47(2): p. 335-42. 
[60] Merrill, D.R., M. Bikson, and J.G. Jefferys, Electrical stimulation of excitable tissue: design of efficacious and safe protocols. J Neurosci Methods, 2005. 141(2): p. 171-98.

[61] Palm, U., et al., The Role of Contact Media at the Skin-electrode Interface during Transcranial Direct Current Stimulation (tDCS). Brain Stimulation, 2014. 7(5): p. 762-764.

[62] Brunoni, A.R., et al., Transcranial direct current stimulation (tDCS) in unipolar vs. bipolar depressive disorder. Prog Neuropsychopharmacol Biol Psychiatry, 2011. 35(1): p. 96-101.

[63] Fertonani, A., C. Ferrari, and C. Miniussi, What do you feel if I apply transcranial electric stimulation? Safety, sensations and secondary induced effects. Clinical Neurophysiology, 2015. 126(11): p. 2181-2188.

[64] Kalu, U.G., et al., Transcranial direct current stimulation in the treatment of major depression: a meta-analysis. Psychological Medicine, 2012. 42(9): p. 1791-1800.

[65] Loo, C.K., et al., Transcranial direct current stimulation for depression: 3-week, randomised, sham-controlled trial. Br J Psychiatry, 2012. 200(1): p. 52-9.

[66] Klem, G.H., et al., The ten-twenty electrode system of the International Federation. The International Federation of Clinical Neurophysiology. Electroencephalogr Clin Neurophysiol Suppl, 1999. 52: p. 3-6.

[67] Oostenveld, R. and P. Praamstra, The five percent electrode system for high-resolution EEG and ERP measurements. Clin Neurophysiol, 2001. 112(4): p. 713-9.

[68] Seibt, O., et al., The Pursuit of DLPFC: Non-neuronavigated Methods to Target the Left Dorsolateral Pre-frontal Cortex With Symmetric Bicephalic Transcranial Direct Current Stimulation (tDCS). Brain Stimul, 2015. 8(3): p. 590-602.

[69] Feurra, M., et al., Frequency-dependent tuning of the human motor system induced by transcranial oscillatory potentials. J Neurosci, 2011. 31(34): p. 12165-70.

[70] Feurra, M., et al., State-Dependent Effects of Transcranial Oscillatory Currents on the Motor System: What You Think Matters. Journal of Neuroscience, 2013. 33(44): p. 17483-17489.

[71] Feurra, M., et al., Frequency specific modulation of human somatosensory cortex. Front Psychol, 2011. 2: p. 13.

[72] Santarnecchi, E., et al., Time Course of Corticospinal Excitability and Autonomic Function Interplay during and Following Monopolar tDCS. Front Psychiatry, 2014. 5: p. 86.

[73] Woods, A.J., et al., A technical guide to tDCS, and related non-invasive brain stimulation tools. Clin Neurophysiol, 2016. 127(2): p. 1031-48.

[74] Miranda, P.C., P. Faria, and M. Hallett, What does the ratio of injected current to electrode area tell us about current density in the brain during tDCS? Clinical Neurophysiology, 2009. 120(6): p. 1183-1187.

[75] Nitsche, M.A., et al., Level of action of cathodal DC polarisation induced inhibition of the human motor cortex. Clin Neurophysiol, 2003. 114(4): p. 600-4.

[76] Nitsche, M.A. and W. Paulus, Sustained excitability elevations induced by transcranial DC motor cortex stimulation in humans. Neurology, 2001. 57(10): p. 1899-901.

[77] Batsikadze, G., et al., Partially non-linear stimulation intensity-dependent effects of direct current stimulation on motor cortex excitability in humans. J Physiol, 2013. 591(7): p. 1987-2000.

[78] Monte-Silva, K., et al., Induction of late LTP-like plasticity in the human motor cortex by repeated non-invasive brain stimulation. Brain Stimul, 2013. 6(3): p. 424-32.

[79] Goldsworthy, M.R., J.B. Pitcher, and M.C. Ridding, Spaced Noninvasive Brain Stimulation: Prospects for Inducing Long-Lasting Human Cortical Plasticity. Neurorehabil Neural Repair, 2015. 29(8): p. 714-21.

[80] Nitsche, M.A., et al., Transcranial direct current stimulation: State of the art 2008. Brain Stimul, 2008. 1(3): p. 206-23.

[81] Poreisz, C., et al., Safety aspects of transcranial direct current stimulation concerning healthy subjects and patients. Brain Res Bull, 2007. 72(4-6): p. 208-14.

[82] Vigano, A., et al., Transcranial Direct Current Stimulation (tDCS) of the visual cortex: a proof-of-concept study based on interictal electrophysiological abnormalities in migraine. J Headache Pain, 2013. 14: p. 23.

[83] Kessler, S.K., et al., Dosage considerations for transcranial direct current stimulation in children: a computational modeling study. PLoS One, 2013. 8(9): p. e76112.

[84] Minhas, P., et al., Transcranial direct current stimulation in pediatric brain: a computational modeling study. Conf Proc IEEE Eng Med Biol Soc, 2012. 2012: p. 859-62.

[85] Wexler, A., The practices of do-it-yourself brain stimulation: implications for ethical considerations and regulatory proposals. J Med Ethics, 2016. 42(4): p. 211-5.

[86] Bhaskar, R.K., et al., Prevalence and correlates of tobacco use among adolescents in the schools of Kalaiya, Nepal: a cross-sectional questionnaire based study. Tobacco Induced Diseases, 2016. 14.

[87] Parajuli, V.J., S. Macdonald, and M. Jimba, Social-Contextual Factors Associated with Alcohol Use among Adolescents of Traditional Alcohol User and Nonuser Ethnic Groups of Nepal. Journal of Ethnicity in Substance Abuse, 2015. 14(2): p. 151-165.

[88] Thapa, N., et al., Alcohol Consumption Practices among Married Women of Reproductive Age in Nepal: A Population Based Household Survey. Plos One, 2016. 11(4).

[89] Shrestha, N.M., Alcohol and drug abuse in Nepal. Br J Addict, 1992. 87(9): p. 1241-8.

[90] Boggio, P.S., et al., Prefrontal cortex modulation using transcranial DC stimulation reduces alcohol craving: A double-blind, sham-controlled study. Drug and Alcohol Dependence, 2008. 92(1-3): p. 55-60.

[91] den Uyl, T.E., T.E. Gladwin, and R.W. Wiers, Transcranial direct current stimulation, implicit alcohol associations and craving. Biol Psychol, 2015. 105: p. 37-42.

[92] Falcone, M., et al., Transcranial Direct Current Brain Stimulation Increases Ability to Resist Smoking. Brain Stimulation, 2016. 9(2): p. 191-196. 
[93] Fregni, F., et al., Cortical stimulation of the prefrontal cortex with transcranial direct current stimulation reduces cue-provoked smoking craving: a randomized, sham-controlled study. J Clin Psychiatry, 2008. 69(1): p.
$32-40$.

[94] Shaik, M.M., K.W. Loo, and S.H. Gan, Burden of stroke in Nepal. Int J Stroke, 2012. 7(6): p. 517-20. 